\title{
LOCAL PROPERTIES OF SOLUTIONS OF ELLIPTIC EQUATIONS DEPENDING ON LOCAL PROPERTIES OF THE DATA*
}

\author{
LUCIO BOCCARDO ${ }^{\dagger}$ AND TOMMASO LEONORI ${ }^{\ddagger}$ \\ Neil: “.. ma misi me per l'alto mare aperto sol con un legno"; \\ qui trovasti "quella compagna picciola da la qual non fui diserto."
}

(Dante: Inferno XXVI)

Abstract. In this paper we deal with local properties of solutions of the boundary value problem

$$
\left\{\begin{aligned}
-\operatorname{div}(a(x, u, \nabla u)) & =\mu & & \text { in } \Omega, \\
u & =0 & & \text { on } \partial \Omega
\end{aligned}\right.
$$

where the left hand side is a Leray-Lions operator and $\mu$ a Radon measure. In particular we look at properties of the solution away from the set where the datum is singular.

Key words. Nonlinear elliptic equations, local estimates, measure data.

AMS subject classifications. 35J65 (35J60, 35J25)

1. Introduction and main results. This paper deals with properties of solutions of nonlinear boundary value problems of the type

$$
\left\{\begin{aligned}
-\operatorname{div}(a(x, u, \nabla u)) & =\mu & & \text { in } \Omega, \\
u & =0 & & \text { on } \partial \Omega
\end{aligned}\right.
$$

or

$$
\left\{\begin{aligned}
-\operatorname{div}(a(x, u, \nabla u)) & =f(x) & & \text { in } \Omega, \\
u & =0 & & \text { on } \partial \Omega
\end{aligned}\right.
$$

where $\Omega$ is a bounded, open subset of $\mathbb{R}^{N}, N>2$, the right hand side is either a bounded Radon measure $\mu$ or a summable function $f$ and the partial differential operator $A$ is defined as

$$
A(v)=-\operatorname{div}(a(x, v, \nabla v))
$$

where $a: \Omega \times \mathbb{R} \times \mathbb{R}^{N} \rightarrow \mathbb{R}^{N}$ is a Carathéodory function (that is, measurable with respect to $x$ in $\Omega$ for every $(s, \xi)$ in $\mathbb{R} \times \mathbb{R}^{N}$, and continuous with respect to $(s, \xi)$ in $\mathbb{R} \times \mathbb{R}^{N}$ for almost every $x$ in $\Omega$ ). We assume that there exist two real positive constants $\alpha$ and $\beta$, such that for almost every $x$ in $\Omega$, for every $s$ in $\mathbb{R}$, for every $\xi$ and $\xi^{\prime}$ in $\mathbb{R}^{N}\left(\xi \neq \xi^{\prime}\right)$,

$$
\begin{gathered}
a(x, s, \xi) \cdot \xi \geq \alpha|\xi|^{2}, \\
|a(x, s, \xi)| \leq \beta|\xi|
\end{gathered}
$$

\footnotetext{
*Received April 5, 2008; accepted for publication June 13, 2008.

${ }^{\dagger}$ Dipartimento di Matematica, Università di Roma 1, Piazza A. Moro 2, 00185 Roma, Italia (boccardo@mat.uniroma1.it).

${ }^{\ddagger}$ CMUC, Departamento de Matemática, Universidade de Coimbra, 3001-454 Coimbra, Portugal (leonori@mat.uc.pt).
} 


$$
\left(a(x, s, \xi)-a\left(x, s, \xi^{\prime}\right)\right) \cdot\left(\xi-\xi^{\prime}\right)>0 .
$$

Under these assumptions, $A$ turns out to be pseudomonotone, and is hence surjective on $W_{0}^{1,2}(\Omega)$ (see $\left.[5]\right)$.

We point out that, for the sake of simplicity, we will restrict ourselves to the case of differential operators defined in $W_{0}^{1,2}(\Omega)$, even though our techniques work also in the $W_{0}^{1, p}(\Omega)$-framework, $p>1$.

Of course, the function $u$ is a solution (solution in the sense of distributions) of (1.1) if $u \in W_{0}^{1,1}(\Omega)$ and if

$$
\int_{\Omega} a(x, u, \nabla u) \cdot \nabla \varphi=\int_{\Omega} \varphi d \mu, \quad \forall \varphi \in C_{0}^{\infty}(\Omega) .
$$

The existence of a distributional solution $u$ of (1.1) and (1.2) has been proved by an approximation procedure (see [2], [3]): $u$ belongs to $W_{0}^{1, q}(\Omega), q<\frac{N}{N-1}$, and is the $W_{0}^{1, q}(\Omega)$-limit of a subsequence of $\left\{u_{n}\right\}$, where $u_{n}$ is a weak solution of the Dirichlet problem

$$
u_{n} \in W_{0}^{1,2}(\Omega):-\operatorname{div}\left(a\left(x, u_{n}, \nabla u_{n}\right)\right)=f_{n}(x)
$$

and

$$
\left\{\begin{array}{c}
\left\{f_{n}\right\} \text { is a sequence of smooth functions } \\
\text { converging to } \mu \text { in } \mathcal{M}(\Omega), \int_{\Omega}\left|f_{n}\right| \text { bounded, }
\end{array}\right.
$$

if we study the boundary value (1.1), and

$$
\left\{\begin{array}{c}
\left\{f_{n}\right\} \text { is a sequence of smooth functions } \\
\text { converging to } f \text { in } L^{1}(\Omega),
\end{array}\right.
$$

if we study the boundary value (1.2).

Thus the results of Theorem 1.1 and Theorem 1.2 concern the solutions $u$ obtained as limit of $u_{n}$, as above.

On the other hand if we consider a datum $f \in L^{m}(\Omega), 1<m<\frac{2 N}{N+2}$, it has been proved in [3] that the sequence $\left\{u_{n}\right\}$ is bounded in $W_{0}^{1, m^{*}}(\Omega)$, so that $u$ belongs to $W_{0}^{1, m^{*}}(\Omega)$, where by $m^{*}=\frac{m N}{N-m}, m<N$, we denote the Sobolev conjugate exponent of $m$.

The properties of local summability for "local solutions" have been studied by G. Stampacchia (see Theorem 5.4 of [8]), if $m>\frac{2 N}{N+2}$.

Here we look at local properties of (global) solutions of (1.1); more precisely we study the behavior of the solutions "far" from the singularities of the datum.

In other words, as the intuition suggests, we expect that a solution of the mentioned problems has suitable summability properties that depend on the local regularity of the datum. For instance, if the datum $f(x)$ (or $\mu$ ) has a singularity concentrated only at a certain $x_{0} \in \Omega$, we expect that the solution is smooth away from $x_{0}$.

We state here our results if the right hand side $f$ belongs to $L^{1}(\Omega)$, whereas $f \psi \in L^{m}(\Omega), m>1$, where the function $\psi$ belongs to $W^{1, \infty}(\Omega)$ : even if $u$ only belongs to $W_{0}^{1, q}(\Omega), q<\frac{N}{N-1}$, the function $u \psi^{\eta}$ is more regular for some $\eta>1$ (its regularity depending on $m$ ).

In the same spirit of the existence results quoted above, the main point in our are a priori estimates on the sequences $\left\{\psi^{\eta} u_{n}\right\},\left\{\psi^{\eta} \nabla u_{n}\right\}$, for some $\eta>1$. 
Our proofs are completely self contained and follow the techniques of [8], [2], [3], [1]. In particular, in Lemma 2.3 we follow the idea of the paper [8] by G. Stampacchia; it would be interesting to give a second proof following the idea of the paper [10] by N. Trudinger.

Let us define, now,

$$
\mathcal{E}_{L}=\left\{\psi \in W^{1, \infty}(\Omega): 0 \leq \psi \leq 1,|\nabla \psi| \leq L\right\} .
$$

Theorem 1.1. Assume (1.3), (1.4), (1.5). Let $f \in L^{1}(\Omega)$ be such that there exists $\psi \in \mathcal{E}_{L}$ with the property $f \psi \in L^{m}(\Omega), 1<m$. Then there exist a distributional solution $u$ of (1.2) and $\eta>1$ (depending on $\psi, m$ and $N$ ) such that

- $u \psi^{\eta} \in W_{0}^{1, m^{*}}(\Omega)$, if $1<m<\frac{2 N}{N+2}$;

- $u \psi^{\eta} \in W_{0}^{1,2}(\Omega) \cap L^{m^{* *}}(\Omega)$, if $\frac{2 N}{N+2} \leq m<\frac{N}{2}$;

- $u \psi^{\eta} \in W_{0}^{1,2}(\Omega) \cap L^{\infty}(\Omega)$, if $m>\frac{N}{2}$.

We also state the following theorem concerning Dirichlet problems with right hand side measure. Thanks to the above considerations, the proof is the same of Theorem 1.1 .

Theorem 1.2. Assume (1.3), (1.4), (1.5). Assume that there exists $\psi \in \mathcal{E}_{L}$ with the property that the sequence $\left\{f_{n} \psi\right\}$ is bounded in $L^{m}(\Omega), 1<m$, where $\left\{f_{n}\right\}$ is the sequence defined in (1.7). Then there exist a distributional solution $u$ of (1.1) and $\eta>1$ (depending on $\psi, m$ and $N$ ) such that

- $u \psi^{\eta} \in W_{0}^{1, m^{*}}(\Omega)$, if $1<m<\frac{2 N}{N+2}$;

- $u \psi^{\eta} \in W_{0}^{1,2}(\Omega) \cap L^{m^{* *}}(\Omega)$, if $\frac{2 N}{N+2} \leq m<\frac{N}{2}$;

- $u \psi^{\eta} \in W_{0}^{1,2}(\Omega) \cap L^{\infty}(\Omega)$, if $m>\frac{N}{2}$.

Note that we prove the results for distributional solutions of (1.1) and (1.2) obtained as limit of approximations. The enhanced regularity of solutions is not true in general since, as a counterexample by J. Serrin shows ([7]), distributional solutions of (1.1) and (1.2) may not be unique. Moreover the conclusion of our theorems are false for the pathological solution of the counterexample by J. Serrin.

In [1] (see also [4]), a notion of solution for (1.2) has been introduced if $f \in L^{1}(\Omega)$ and the function $a(x, s, \xi)$ does not depend on $s$, so that the differential operator $A$ is strictly monotone, with the purpose of proving its uniqueness: the so-called entropy solution. In this case, the strong limit $u$ in $W_{0}^{1, q}(\Omega), q<\frac{N}{N-1}$, of the sequence $\left\{u_{n}\right\}$ is the unique entropy solution of (1.2), so that Theorem 1.1 can be seen as giving improved summability properties of the entropy solution.

2. A priori estimates and proof of the results. We begin recalling the following proposition (see [2]).

Proposition 2.1. The sequence $\left\{u_{n}\right\}$ of solutions of (1.6) is bounded in $L^{\sigma}(\Omega)$, for every $\sigma<\frac{N}{N-2}$.

The first step relies in proving some local summability properties of the sequence $\left\{u_{n}\right\}$.

Lemma 2.2. Assume (1.3), (1.4), (1.5) and that $\left\{f_{n} \psi\right\}$ is bounded in $L^{m}(\Omega)$, $1<m<\frac{N}{2}$, where $\left\{f_{n}\right\}$ is as in (1.8) and $\psi \in \mathcal{E}_{L}$. Then there exists $\eta_{1}>1$ (depending on $\psi, m$ and $N$ ) such that the sequence $\left\{u_{n} \psi^{\eta_{1}}\right\}$ is bounded in $L^{m^{* *}}(\Omega)$. 
Proof. We follow [3] and we choose $v_{\epsilon} \psi^{p}$ as test function in (1.6), where $v_{\epsilon}$ is the function $\left[\left(\epsilon+\left|u_{n}\right|\right)^{2 \gamma-1}-\epsilon^{2 \gamma-1}\right] \operatorname{sgn}\left(u_{n}\right), p=2+2 \gamma, \gamma \in\left(\frac{1}{2}, \frac{m^{* *}}{2^{*}}\right)$. Note that $\frac{1}{2}<\frac{m^{* *}}{2^{*}}$ since $m>1$. Moreover since $f_{n} \in L^{\infty}(\Omega)$, then every $u_{n}$ is a bounded function, so that $v_{\epsilon}$ belongs to $W_{0}^{1,2}(\Omega) \cap L^{\infty}(\Omega)$. Hence, by (1.3) and (1.4), we have

$$
\begin{gathered}
\alpha(2 \gamma-1) \int_{\Omega} \psi^{p}\left|\nabla u_{n}\right|^{2}\left(\epsilon+\left|u_{n}\right|\right)^{2(\gamma-1)} \leq \int_{\Omega}\left(f_{n} \psi\right)\left(v_{\epsilon} \psi^{p-1}\right) \\
+p \beta \int_{\Omega} \psi^{p}\left|\nabla u_{n}\right||\nabla \psi|\left[\left(\epsilon+\left|u_{n}\right|\right)^{(2 \gamma-1)}-\epsilon^{(2 \gamma-1)}\right],
\end{gathered}
$$

and by Young's inequality we get

$$
\begin{gathered}
\int_{\Omega} \psi^{p}\left|\nabla u_{n}\right|^{2}\left(\epsilon+\left|u_{n}\right|\right)^{2(\gamma-1)} \leq \frac{2\left\|f_{n} \psi\right\|_{m}}{\alpha(2 \gamma-1)}\left(\int_{\Omega}\left|v_{\epsilon}\right|^{m^{\prime}} \psi^{(p-1) m^{\prime}}\right)^{\frac{1}{m^{\prime}}} \\
+\frac{p^{2} \beta^{2} L^{2}}{\alpha^{2}(2 \gamma-1)^{2}} \int_{\Omega} \psi^{p-2}\left(\epsilon+\left|u_{n}\right|\right)^{2 \gamma}
\end{gathered}
$$

We note that

$$
\begin{gathered}
\left|\nabla\left\{\left[\left(\epsilon+\left|u_{n}\right|\right)^{\gamma}-\epsilon^{\gamma}\right] \psi^{\frac{p}{2}}\right\}\right|^{2} \\
\leq 2 \gamma^{2} \psi^{p}\left|\nabla u_{n}\right|^{2}\left(\epsilon+\left|u_{n}\right|\right)^{2(\gamma-1)}+\frac{p^{2}}{2} \psi^{p-2} L^{2}\left[\left(\epsilon+\left|u_{n}\right|\right)^{\gamma}-\epsilon^{\gamma}\right]^{2},
\end{gathered}
$$

so that by Sobolev inequality ( $\mathcal{S}$ denotes the Sobolev constant) we have

$$
\begin{gathered}
\mathcal{S}^{2}\left(\int_{\Omega}\left\{\left[\left(\epsilon+\left|u_{n}\right|\right)^{\gamma}-\epsilon^{\gamma}\right] \psi^{\frac{p}{2}}\right\}^{2^{*}}\right)^{\frac{2}{2^{*}}} \leq \int_{\Omega}\left|\nabla\left\{\left[\left(\epsilon+\left|u_{n}\right|\right)^{\gamma}-\epsilon^{\gamma}\right] \psi^{\frac{p}{2}}\right\}\right|^{2} \\
\leq 2 \gamma^{2} \frac{2\left\|f_{n} \psi\right\|_{m}}{\alpha(2 \gamma-1)}\left(\int_{\Omega}\left|v_{\epsilon}\right|^{m^{\prime}} \psi^{(p-1) m^{\prime}}\right)^{\frac{1}{m^{\prime}}} \\
+\left[\frac{2 \gamma^{2} p^{2} \beta^{2}}{\alpha^{2}(2 \gamma-1)^{2}}+\frac{p^{2}}{2}\right] L^{2} \int_{\Omega} \psi^{p-2}\left(\epsilon+\left|u_{n}\right|\right)^{2 \gamma}
\end{gathered}
$$

Now, taking the limit as $\epsilon \rightarrow 0$ and using that $\gamma<\frac{m^{* *}}{2^{*}}$ (and consequently that $\left.(2 \gamma-1) m^{\prime}<\gamma 2^{*}\right)$ we get

$$
\begin{gathered}
\mathcal{S}^{2}\left(\int_{\Omega}\left|u_{n}\right|^{2^{*} \gamma} \psi^{\frac{p 2^{*}}{2}}\right)^{\frac{2}{2^{*}}} \\
\leq \frac{4 \gamma^{2}\left\|f_{n} \psi\right\|_{m}}{\alpha(2 \gamma-1)}\left(\int_{\Omega}\left|u_{n}\right|^{(2 \gamma-1) m^{\prime}} \psi^{(p-1) m^{\prime}}\right)^{\frac{1}{m^{\prime}}} \\
+\left[\frac{2 \gamma^{2} p^{2} \beta^{2}}{\alpha^{2}(2 \gamma-1)^{2}}+\frac{p^{2}}{2}\right] L^{2} \int_{\Omega} \psi^{p-2}\left|u_{n}\right|^{2 \gamma} \\
\leq \frac{4 \gamma^{2}\left\|f_{n} \psi\right\|_{m}|\Omega|^{\gamma^{*}-(2 \gamma-1) m^{\prime}}}{\alpha(2 \gamma-1)}\left(\int_{\Omega}\left|u_{n}\right|^{\gamma 2^{*} m^{\prime}} \psi^{\frac{(p-1) \gamma^{*}}{(2 \gamma-1)}}\right)^{\frac{(2 \gamma-1)}{\gamma^{*}}} \\
+\left[\frac{2 \gamma^{2} p^{2} \beta^{2}}{\alpha^{2}(2 \gamma-1)^{2}}+\frac{p^{2}}{2}\right] L^{2} \int_{\Omega} \psi^{p-2}\left|u_{n}\right|^{2 \gamma} .
\end{gathered}
$$


Note that $\frac{(p-1) \gamma 2^{*}}{(2 \gamma-1)}>\frac{p 2^{*}}{2}$ since $p>2 \gamma$, so we apply Young's inequality in the first term on the right hand side of the previous inequality (with exponents $\frac{2 \gamma}{2 \gamma-1}$ and $2 \gamma$ ) and we have

$$
\begin{gathered}
\mathcal{S}^{2}\left(\int_{\Omega}\left|u_{n}\right|^{2^{*} \gamma} \psi^{\frac{p 2^{*}}{2}}\right)^{\frac{2}{2^{*}}} \\
\leq \frac{\mathcal{S}^{2}}{2}\left(\int_{\Omega}\left|u_{n}\right|^{\gamma 2^{*}} \psi^{\frac{p 2^{*}}{2}}\right)^{\frac{2}{2 *}}+\frac{\mathcal{S}^{2}}{2(2 \gamma-1)}\left[\frac{4 \gamma\left\|f_{n} \psi\right\|_{m}}{\alpha \mathcal{S}^{2}}|\Omega|^{\frac{\gamma 2^{*}-(2 \gamma-1) m^{\prime}}{\gamma 2^{*} m^{\prime}}}\right]^{2 \gamma} \\
+\left[\frac{2 \gamma^{2} p^{2} \beta^{2}}{\alpha^{2}(2 \gamma-1)^{2}}+\frac{p^{2}}{2}\right] L^{2} \int_{\Omega} \psi^{p-2}\left|u_{n}\right|^{2 \gamma} .
\end{gathered}
$$

Setting

$$
C_{1}^{\gamma}=\frac{1}{2 \gamma-1}\left[\frac{4 \gamma|\Omega|^{\frac{\gamma 2^{*}-(2 \gamma-1) m^{\prime}}{\gamma 2^{*} m^{\prime}}}}{\alpha \mathcal{S}^{2}}\right]^{2 \gamma} \text { and } C_{2}^{\gamma}=2\left[\frac{2 \gamma^{2} p^{2} \beta^{2}}{\alpha^{2}(2 \gamma-1)^{2}}+\frac{p^{2}}{2}\right] \frac{L^{2}}{\mathcal{S}^{2}}
$$

we get

$$
\left(\left.\int_{\Omega}\left|u_{n}\left[\left.\psi^{\left.\frac{p-2}{2 \gamma}\right]^{\frac{p}{p-2}}}\right|^{2^{*} \gamma}\right)^{\frac{2}{2^{*}}} \leq C_{1}^{\gamma}\left\|f_{n} \psi\right\|_{m}^{2 \gamma}+C_{2}^{\gamma} \int_{\Omega}\right| u_{n} \psi^{\frac{p-2}{2 \gamma}}\right|^{2 \gamma} .\right.
$$

Note that both $C_{1}^{\gamma}$ and $C_{2}^{\gamma}$ blow-up for $\gamma=\frac{1}{2}$; nevertheless, as we will see later, we will need to deal with values of $\gamma>\gamma_{0}>\frac{1}{2}$.

Recalling that $p=2+2 \gamma$ the previous inequality becomes

$$
\int_{\Omega}\left|u_{n}[\psi]^{1+\frac{1}{\gamma}}\right|^{2^{*} \gamma} \leq C_{0}\left[\left\|f_{n} \psi\right\|_{m}^{2^{*} \gamma}+\left(\int_{\Omega}\left|u_{n} \psi\right|^{2 \gamma}\right)^{\frac{2^{*}}{2}}\right],
$$

where $C_{0}=\max \left\{\frac{\left(2 C_{1}^{\gamma}\right)^{\frac{N}{N-2}}}{2}, \frac{\left(2 C_{2}^{\gamma}\right)^{\frac{N}{N-2}}}{2}, 1\right\}$.

It is clear that inequality (2.4) is crucial in order to obtain the result: indeed it is, roughly speaking, a control of the weighted norm of $u_{n}$ in a Lebesgue space with a norm in a bigger Lebesgue space, but with a different weight.

Note that the previous inequality, thanks to Proposition 2.1, implies the result for "small" $m: 1<m<\frac{N^{2}}{N^{2}-2 N+4}$, that is $m$ such that $m^{* *}<\left(\frac{2^{*}}{2}\right)^{2}$.

In the case $m^{* *} \geq\left(\frac{2^{*}}{2}\right)^{2}$, the idea of our proof is to use (2.4) recursively a finite number of times; therefore we do not need a precise control on the quantities in the left hand side of (2.4).

Recalling the result of Proposition 2.1, our starting point is $I \in \mathbb{N}, I \geq 1$, such that $2 \frac{m^{* *}}{2^{*}} \frac{2^{I}}{\left(2^{*}\right)^{I}}<\frac{2^{*}}{2}$, i.e.

$$
I=\min \left\{j \in \mathbb{N}: 2 \frac{m^{* *}}{2^{*}}\left(\frac{2}{2^{*}}\right)^{j}<\frac{N}{N-2}\right\}=\min \left\{j \in \mathbb{N}: m^{* *}<\left(\frac{2^{*}}{2}\right)^{j+2}\right\} .
$$

Moreover we define

$$
\gamma_{i}=\frac{m^{* *}}{2^{*}}\left(\frac{2}{2^{*}}\right)^{I-i}, \quad 0 \leq i \leq I,
$$


and we note that

$$
\frac{1}{2}<\frac{m^{* *}}{2^{*}}\left(\frac{2}{2^{*}}\right)^{I}=\gamma_{0} \leq \gamma_{i} \leq \gamma_{I}=\frac{m^{* *}}{2^{*}},
$$

so that our constants, which depend continuously on $\gamma_{i}$, will run on a bounded subset of $\mathbb{R}$.

If $I=1$, we consider only the first inequality below $\left(I \geq 1\right.$ since $\left.m^{* *} \geq\left(\frac{2^{*}}{2}\right)^{2}\right)$; while, if $I \geq 2$, let us define $\psi_{i+1}=\psi_{i}^{1+\frac{1}{\gamma_{i}}}, i \geq 1 ; \psi_{0}=\psi$. Note that $2 \gamma_{i}=2^{*} \gamma_{i-1}$ and that $\psi_{i} \leq \psi_{i-1}$, so that, $\forall i \in[1, I]$,

$$
\begin{gathered}
\int_{\Omega}\left|u_{n} \psi_{i}\right|^{2 \gamma_{i}}=\int_{\Omega}\left|u_{n} \psi_{i}\right|^{2^{*} \gamma_{i-1}} \leq C_{0}\left[\left\|f_{n} \psi_{i-1}\right\|_{m}^{2^{*} \gamma_{i-1}}+\left(\int_{\Omega}\left|u_{n} \psi_{i-1}\right|^{2 \gamma_{i-1}}\right)^{\frac{2^{*}}{2}}\right] \\
=C_{0}\left[\left\|f_{n} \psi_{i-1}\right\|_{m}^{2 \gamma_{i}}+\left(\int_{\Omega}\left|u_{n} \psi_{i-1}\right|^{2^{*} \gamma_{i-2}}\right)^{\frac{2^{*}}{2}}\right] \\
\leq C_{0}\left[\left\|f_{n} \psi_{i-1}\right\|_{m}^{2^{*} \gamma_{i-1}}+2^{\frac{2^{*}}{2}} C_{0}^{\frac{2^{*}}{2}}\left[\left\|f_{n} \psi_{i-2}\right\|_{m}^{2^{*}}\left(2^{*} \gamma_{i-2}\right)+\left(\int_{\Omega}\left|u_{n} \psi_{i-2}\right|^{2 \gamma_{i-2}}\right)^{\left(\frac{2^{*}}{2}\right)^{2}}\right]\right] \\
\leq 2^{\frac{2^{*}}{2}} C_{0}^{\frac{2^{*}}{2}+1}\left[\left\|f_{n} \psi_{i-1}\right\|_{m}^{2 \gamma_{i}}+\left\|f_{n} \psi_{i-2}\right\|_{m}^{2 \gamma_{i}}+\left(\int_{\Omega}\left|u_{n} \psi_{i-2}\right|^{2 \gamma_{i-2}}\right)^{\left(\frac{2^{*}}{2}\right)^{2}}\right],
\end{gathered}
$$

Thus setting $i=I$ and iterating this inequality we deduce that there exist two constants $C_{f}$ and $C_{2}$ such that

$$
\int_{\Omega}\left|u_{n} \psi_{I}\right|^{2 \gamma_{I}} \leq C_{f}+C_{3}\left(\int_{\Omega}\left|u_{n} \psi_{0}\right|^{2 \gamma_{0}}\right)^{\left(\frac{2^{*}}{2}\right)^{I}},
$$

where

$$
C_{3}=C_{0}\left(2 C_{0}\right)^{\sum_{k=1}^{I}\left(\frac{2 *}{2}\right)^{k}} \quad \text { and } \quad C_{f}=C_{3}\left(\sum_{j=1}^{I}\left\|f_{n} \psi_{I-j}\right\|_{m}^{2 \gamma_{I}}\right)
$$

using again inequality (2.4) we obtain

$$
\begin{gathered}
\left.\int_{\Omega}\left|u_{n}\left[\psi_{I}\right]^{1+\left.\frac{1}{\gamma_{I}}\right|^{m^{* *}}}=\int_{\Omega}\right| u_{n}\left[\psi_{I}\right]^{1+\frac{1}{\gamma_{I}}}\right|^{2^{*} \gamma_{I}} \\
\leq C\left[\left\|f_{n} \psi_{I}\right\|_{m}^{2 \gamma_{I}}+\left(\int_{\Omega}\left|u_{n} \psi_{I}\right|^{2 \gamma_{I}}\right)^{\frac{2}{2^{*}}}\right] .
\end{gathered}
$$

Combining the above inequality with (2.5), using that $2 \gamma_{0}<\frac{N}{N-2}$ and the result of Proposition 2.1, we deduce that there exist $\eta_{1}>0$ and $M>0$ such that

$$
\left\|u_{n} \psi^{\eta_{1}}\right\|_{m^{* *}} \leq M, \quad \text { where } \quad \eta_{1}=\prod_{i=0}^{I}\left(1+\frac{1}{\gamma_{i}}\right) .
$$

Now we prove a local boundedness property for the sequence $\left\{u_{n}\right\}$. 
Lemma 2.3. Assume (1.3), (1.4), (1.5), (1.8) and that $\left\{f_{n} \psi\right\}$ is bounded in $L^{m}(\Omega), m>\frac{N}{2}$, where $\left\{f_{n}\right\}$ is as in (1.8) and $\psi \in \mathcal{E}_{L}$. Then there exists $\eta_{1}>1$ (depending on $\psi$ and $N$ ) such that the sequence $\left\{u_{n} \psi^{1+\eta_{1}}\right\}$ is bounded in $L^{\infty}(\Omega)$.

Proof. We first note that by Lemma 2.2, since $\left\{f_{n} \psi\right\}$ is bounded also in $L^{s}(\Omega)$, $\forall s \leq \frac{N}{2}$, then for every fixed $r>1$, there exists $\eta>1$ such that $\left\{u_{n} \psi^{\eta}\right\}$ is bounded in $L^{r}(\Omega)$ (even though not uniformly with respect to $r$ ). Following G. Stampacchia (see $[8]$ ), we define

$$
G_{k}(s)= \begin{cases}s+k, & \text { if } s \leq-k \\ 0, & \text { if }-k \leq s<k \\ s-k, & \text { if } s \geq k\end{cases}
$$

and

$$
A_{k}=\left\{x \in \Omega:\left|u_{n} \psi^{p}\right| \geq k\right\} .
$$

Let us multiply the equation (1.6) by $\psi^{p} G_{k}\left(u_{n} \psi^{p}\right)$, where $p>1$ is to be chosen. Thus

$$
\begin{gathered}
p \int_{A_{k}} a\left(x, u_{n}, \nabla u_{n}\right) \cdot \nabla \psi \psi^{p-1} G_{k}\left(u_{n} \psi^{p}\right) \\
+p \int_{A_{k}} a\left(x, u_{n}, \nabla u_{n}\right) \cdot \nabla \psi \psi^{2 p-1} u_{n} \\
+\int_{A_{k}} a\left(x, u_{n}, \nabla u_{n}\right) \cdot \nabla u_{n} \psi^{2 p}=\int_{A_{k}} f_{n} \psi^{p} G_{k}\left(u_{n} \psi^{p}\right) .
\end{gathered}
$$

By (1.4) and Young's inequality, we deduce

$$
\begin{gathered}
p \int_{A_{k}}\left|a\left(x, u_{n}, \nabla u_{n}\right) \cdot \nabla \psi \psi^{2 p-1} u_{n}\right| \\
\leq \frac{\alpha}{4} \int_{A_{k}}\left|\nabla u_{n}\right|^{2} \psi^{2 p}+\frac{p^{2} \beta^{2}}{\alpha} \int_{A_{k}}|\nabla \psi|^{2} \psi^{2(p-1)}\left|u_{n}\right|^{2} .
\end{gathered}
$$

Moreover since $\left|G_{k}(s)\right| \leq|s|, \forall k>0, \forall s \in \mathbb{R}$, we have

$$
\begin{gathered}
p \int_{A_{k}}\left|a\left(x, u_{n}, \nabla u_{n}\right) \cdot \nabla \psi \psi^{p-1} G_{k}\left(u_{n} \psi^{p}\right)\right| \\
\leq p \int_{A_{k}}\left|a\left(x, u_{n}, \nabla u_{n}\right) \cdot \nabla \psi\right|\left|u_{n}\right| \psi^{2 p-1} \\
\leq \frac{\alpha}{4} \int_{A_{k}}\left|\nabla u_{n}\right|^{2} \psi^{2 p}+\frac{\beta^{2} p^{2}}{\alpha} \int_{A_{k}}|\nabla \psi|^{2} \psi^{2(p-1)}\left|u_{n}\right|^{2} .
\end{gathered}
$$

Thus, by (1.3), by adding $\frac{\alpha}{2} \int_{A_{k}}\left|u_{n}\right|^{2}\left|\nabla \psi^{p}\right|^{2}$ on both sides of (2.7) and by the previous inequalities, we get

$$
\begin{gathered}
\frac{\alpha}{2} \int_{A_{k}}\left|\nabla u_{n}\right|^{2} \psi^{2 p}+\frac{\alpha}{2} \int_{A_{k}}\left|u_{n}\right|^{2}\left|\nabla \psi^{p}\right|^{2} \\
\leq \int_{A_{k}} f_{n} \psi^{p} G_{k}\left(u_{n} \psi^{p}\right)+\left(2 \frac{\beta^{2} p^{2}}{\alpha}+\frac{\alpha p^{2}}{2}\right) \int_{A_{k}}|\nabla \psi|^{2} \psi^{2(p-1)}\left|u_{n}\right|^{2} .
\end{gathered}
$$


Since $G_{k}\left(u_{n} \psi^{p}\right) \in W_{0}^{1,2}(\Omega)$ we deduce by Sobolev inequality

$$
\begin{gathered}
\mathcal{S}^{2} \frac{\alpha}{4}\left(\int_{\Omega}\left|G_{k}\left(u_{n} \psi^{p}\right)\right|^{2^{*}}\right)^{\frac{2}{2^{*}}} \leq \frac{\alpha}{4} \int_{\Omega}\left|\nabla G_{k}\left(u_{n} \psi^{p}\right)\right|^{2} \\
\leq \int_{A_{k}} f_{n} \psi^{p} G_{k}\left(u_{n} \psi^{p}\right)+\left(2 \frac{\beta^{2} p^{2}}{\alpha}+\frac{\alpha p^{2}}{2}\right) L^{2} \int_{A_{k}} \psi^{2(p-1)}\left|u_{n}\right|^{2} .
\end{gathered}
$$

Moreover we fix $r>N>2$, so that

$$
\int_{A_{k}} \psi^{2(p-1)}\left|u_{n}\right|^{2} \leq\left(\int_{A_{k}}\left(\psi^{p-1}\left|u_{n}\right|\right)^{r}\right)^{\frac{2}{r}}\left|A_{k}\right|^{1-\frac{2}{r}} .
$$

Choosing, now, $p=1+\eta_{1}\left(\eta_{1}\right.$ has been defined in (2.6)) and setting $\left\|u_{n} \psi^{\eta_{1}}\right\|_{r}=R$, we obtain, using (2.6),

$$
\int_{A_{k}} \psi^{2 \eta_{1}}\left|u_{n}\right|^{2} \leq R^{2}\left|A_{k}\right|^{1-\frac{2}{r}}
$$

On the other hand, by Hölder and Young inequalities, we deduce

$$
\int_{A_{k}} f_{n} \psi^{p} G_{k}\left(u_{n} \psi^{p}\right) \leq \frac{2}{\mathcal{S}^{2} \alpha}\left(\int_{A_{k}}\left|\psi^{p} f_{n}\right|^{\frac{2 N}{N+2}}\right)^{\frac{N+2}{N}}+\mathcal{S}^{2} \frac{\alpha}{8}\left(\int_{A_{k}}\left|G_{k}\left(u_{n} \psi^{p}\right)\right|^{2^{*}}\right)^{\frac{2}{2^{*}}} .
$$

Thus the first integral in the right hand side of (2.8) can be absorbed in the left hand side of (2.8), while again by Hölder inequality (with exponents $\frac{m(N+2)}{2 N}$ and $\left.\frac{m(N+2)}{m(N+2)-2 N}\right)$ we get

$$
\begin{gathered}
\mathcal{S}^{2} \frac{\alpha}{8}\left(\int_{\Omega}\left|G_{k}\left(u_{n} \psi^{p}\right)\right|^{2^{*}}\right)^{\frac{2}{2^{*}}} \\
\leq \frac{2}{\mathcal{S}^{2} \alpha}\left(\int_{A_{k}}\left|\psi^{p} f_{n}\right|^{m}\right)^{\frac{2}{m}}\left|A_{k}\right|^{\frac{m(N+2)-2 N}{m N}}+\left(2 \frac{\beta^{2} p^{2}}{\alpha}+\frac{\alpha p^{2}}{2}\right) L^{2} R^{2}\left|A_{k}\right|^{1-\frac{2}{r}} .
\end{gathered}
$$

Now for every $h>k$, there exists $C=C(R)$ such that

$$
(h-k)^{2}\left|A_{h}\right|^{\frac{2}{2^{*}}} \leq C(R)\left(\left|A_{k}\right|^{\frac{m(N+2)-2 N}{m N}}+\left|A_{k}\right|^{1-\frac{2}{r}}\right) .
$$

Moreover, since $r>N$ and $m>\frac{N}{2}$, for $k$ large enough so that $\left|A_{k}\right|<1$ we have

$$
(h-k)^{2}\left|A_{h}\right|^{\frac{2}{2^{*}}} \leq 2 C(R)\left|A_{k}\right|^{\frac{m(N+2)-2 N}{m N}}
$$

which implies

$$
\left|A_{h}\right| \leq C_{1}(R) \frac{\left|A_{k}\right|^{\frac{m(N+2)-2 N}{m(N-2)}}}{(h-k)^{2^{*}}} .
$$


Since $m>\frac{N}{2}$, we have $\frac{m(N+2)-2 N}{m(N-2)}>1$. Thus, thanks to Real Analysis lemma by G. Stampacchia (see [8], Lemma 4.1, first part) there exists $t>0$ such that

$$
\left|A_{t}\right|=0
$$

Hence

$$
\left\|u_{n} \psi^{1+\eta_{1}}\right\|_{\infty} \leq C\left(\left\|f_{n} \psi\right\|_{m}\right)
$$

REMARK 2.4. If in Theorems (1.1) and (1.2) we assume that $m=\frac{N}{2}$, we can deduce on $u \psi^{\eta}$ an exponential summability result thanks to inequality (2.9) and the second part of Lemma 4.1 of [8].

REMARK 2.5. If the datum $f \in L^{1}(\Omega)$ of Theorem 1.2 is such that there exists $\psi \in \mathcal{E}_{L}$ with the property that $f \psi$ belongs to the Marcinkiewicz space $M^{m}(\Omega), 1<m$, then there exist a distributional solution $u$ of (1.2) and $\eta>1$ (depending on $\psi, m$ and $N$ ) such that the conclusion of Theorem 1.2 holds changing Lebesgue spaces with Marcinkiewicz spaces. Indeed, the proof of the previous lemma still holds with the use of Hölder inequality for Marcinkiewicz spaces. Moreover inequality (2.9) and the third part of Lemma 4.1 of [8] imply that $\left\{u_{n} \psi^{1+\eta_{1}}\right\}$ is bounded in the Marcinkiewicz space $M^{m^{* *}}(\Omega)$, since $\frac{2^{*}}{1-\frac{m(N+2)-2 N}{m(N-2)}}=m^{* *}$.

The next two lemmas give local estimates on the sequence $\left\{\nabla u_{n}\right\}$.

LEMma 2.6. Assume (1.3), (1.4), (1.5), (1.8) and that $\left\{f_{n} \psi\right\}$ is bounded in $L^{m}(\Omega), 1<m<\frac{2 N}{N+2}$, where $\left\{f_{n}\right\}$ is as in (1.8) and $\psi \in \mathcal{E}_{L}$. Then there exists $\eta_{2}>1$ such that the sequence $\left\{\psi^{\eta_{2}}\left|\nabla u_{n}\right|^{m^{*}}\right\}$ is bounded in $L^{1}(\Omega)$.

Proof. Arguing as in the first part of the proof of Lemma 2.2 we deduce the analogue of inequality (2.1) with $\gamma=\frac{m^{* *}}{2^{*}}$ (note that now $\frac{1}{2}<\gamma<1$ ): hence $(2 \gamma-1) m^{\prime}=$ $m^{* *}$, and $2 \gamma<(2 \gamma-1) m^{\prime}$. Moreover we set $\eta_{2}=\max \left\{\eta_{1} \frac{m^{* *}}{m^{\prime}}+1, \eta_{1} \frac{2 m^{* *}}{2^{*}}+2, \eta_{1} m^{* *}\right\}$, where $\eta_{1}$ has been defined in (2.6), so that we have (we use Hölder inequality with exponents $\frac{2^{*}}{2}=\frac{m^{* *}}{2 \gamma}$ and $\frac{N}{2}$ )

$$
\begin{gathered}
\int_{\Omega} \psi^{\eta_{2}} \frac{\left|\nabla u_{n}\right|^{2}}{\left(\epsilon+\left|u_{n}\right|\right)^{2(1-\gamma)}} \leq \frac{2\left\|f_{n} \psi\right\|_{m}}{\alpha(2 \gamma-1)}\left(\int_{\Omega}\left|v_{\epsilon}\right|^{m^{\prime}} \psi^{\left(\eta_{2}-1\right) m^{\prime}}\right)^{\frac{1}{m^{\prime}}} \\
+\frac{\eta_{2}^{2} \beta^{2} L^{2}}{\alpha^{2}(2 \gamma-1)^{2}} \int_{\Omega}\left(\epsilon+\left|u_{n}\right|\right)^{2 \gamma} \psi^{\eta_{2}-2} \\
\leq \frac{2\left\|f_{n} \psi\right\|_{m}}{\alpha(2 \gamma-1)}\left(\int_{\Omega}\left(\epsilon+\left|u_{n}\right|\right)^{m^{* *}} \psi^{\left(\eta_{2}-1\right) m^{\prime}}\right)^{\frac{1}{m^{\prime}}} \\
+\frac{\eta_{2}^{2} \beta^{2} L^{2}}{\alpha^{2}(2 \gamma-1)^{2}}|\Omega|^{\frac{2}{N}}\left(\int_{\Omega}\left(\epsilon+\left|u_{n}\right|\right)^{m^{* *}} \psi^{\frac{\left(\eta_{2}-2\right) 2^{*}}{2}}\right)^{\frac{2}{2^{*}}}
\end{gathered}
$$

Note that, since $m<\frac{2 N}{N+2}$, then $m^{*}<2$. Moreover $\frac{2 m^{*}(1-\gamma)}{2-m^{*}}=m^{* *}$. Thus by Hölder 
inequality, using $(2.11)$ and $1-\frac{m^{*}}{2}>0\left(\right.$ since $\left.m<\frac{2 N}{N+2}\right)$, we deduce

$$
\begin{gathered}
\int_{\Omega} \psi^{\eta_{2}}\left|\nabla u_{n}\right|^{m^{*}}=\int_{\Omega} \psi^{\frac{\eta_{2} m^{*}}{2}} \frac{\left|\nabla u_{n}\right|^{m^{*}}}{\left(\epsilon+\left|u_{n}\right|\right)^{(1-\gamma) m^{*}}}\left(\epsilon+\left|u_{n}\right|\right)^{(1-\gamma) m^{*}} \psi^{\eta_{2}\left(1-\frac{m^{*}}{2}\right)} \\
\leq\left[\int_{\Omega} \psi^{\eta_{2}} \frac{\left|\nabla u_{n}\right|^{2}}{\left(\epsilon+\left|u_{n}\right|\right)^{2(1-\gamma)}}\right]^{\frac{m^{*}}{2}}\left[\int_{\Omega}\left(\epsilon+\left|u_{n}\right|\right)^{\frac{2 m^{*}(1-\gamma)}{2-m^{*}}} \psi^{\eta_{2}}\right]^{\frac{2-m^{*}}{2}} \\
\leq\left[\frac{2\left\|f_{n} \psi\right\|_{m}}{\alpha(2 \gamma-1)}\left(\int_{\Omega}\left(\epsilon+\left|u_{n}\right|\right)^{m^{* *}} \psi^{\left(\eta_{2}-1\right) m^{\prime}}\right)^{\frac{1}{m^{\prime}}}\right. \\
\left.+\frac{\eta_{2}^{2} \beta^{2} L^{2}|\Omega| \frac{2}{N}}{\alpha^{2}(2 \gamma-1)^{2}}\left(\int_{\Omega}\left(\epsilon+\left|u_{n}\right|\right)^{m^{* *}} \psi^{\frac{\left(\eta_{2}-2\right) 2^{*}}{2}}\right)^{\frac{2}{2^{*}}}\right]^{\frac{m^{*}}{2}}\left[\int_{\Omega}\left(\epsilon+\left|u_{n}\right|\right)^{\frac{2 m^{*}(1-\gamma)}{2-m^{*}}} \psi^{\eta_{2}}\right]^{\frac{2-m^{*}}{2}} .
\end{gathered}
$$

Letting now $\epsilon$ go to 0 and recalling the choice of $\eta_{2}$, we deduce that

$$
\begin{gathered}
\int_{\Omega} \psi^{\eta_{2}}\left|\nabla u_{n}\right|^{m^{*}} \leq\left[\frac{2\left\|f_{n} \psi\right\|_{m}}{\alpha(2 \gamma-1)}\left(\int_{\Omega}\left(\left|u_{n}\right| \psi^{\eta_{1}}\right)^{m^{* *}}\right)^{\frac{1}{m^{*}}}\right. \\
\left.+\frac{\eta_{2}^{2} \beta^{2} L^{2}}{\alpha^{2}(2 \gamma-1)^{2}}|\Omega|^{\frac{2}{N}}\left(\int_{\Omega}\left(\left|u_{n}\right| \psi^{\eta_{1}}\right)^{m^{* *}}\right)^{\frac{2}{2^{*}}}\right]^{\frac{m^{*}}{2}}\left[\int_{\Omega}\left(\left|u_{n}\right| \psi^{\eta_{1}}\right)^{m^{* *}}\right]^{\frac{2-m^{*}}{2}} .
\end{gathered}
$$

Using Lemma 2.2, the right hand side of the previous inequality is bounded and so the proof is completed.

Lemma 2.7. Assume (1.3), (1.4), (1.5), (1.8) and that $\left\{f_{n} \psi\right\}$ is bounded in $L^{m}(\Omega), m \geq \frac{2 N}{N+2}$, where $\left\{f_{n}\right\}$ is as in (1.8) and $\psi \in \mathcal{E}_{L}$. Then there exists $\eta_{3}>1$ (depending on $\psi, m$ and $N$ ) such that the sequence $\left\{\psi^{\eta_{3}}\left|\nabla u_{n}\right|^{2}\right\}$ is bounded in $L^{1}(\Omega)$.

Proof. Using Lemma 2.2 and $m \geq \frac{2 N}{N+2}$, we can multiply equation (1.6) by $u_{n} \psi^{2\left(\eta_{1}+1\right)}\left(\eta_{1}\right.$ as in (2.6)) and thus, using that $m^{* *} \geq m^{\prime}$, we deduce by standard computations that

$$
\int_{\Omega} \psi^{\eta_{3}}\left|\nabla u_{n}\right|^{2} \leq\left\|f_{n} \psi\right\|_{m}\left\|u_{n} \psi^{\eta_{1}}\right\|_{m^{* *}}^{\frac{m^{* *}}{m^{\prime}}}+2 \frac{\left(\eta_{1}+1\right)^{2} \beta^{2} L^{2}}{\alpha} \int_{\Omega}\left(\left|u_{n}\right| \psi^{\eta_{1}}\right)^{2} .
$$

Therefore, the result holds with $\eta_{3}=2\left(\eta_{1}+1\right)$. $\square$

Now we can prove Theorem 1.1 and Theorem 1.2.

Proof. As already remarked, the existence of a solution for (1.2) and (1.1) is a consequence of compactness results for $u_{n}$ (see [2], [3], [1], [4]), thanks to the properties of the sequence $\left\{f_{n}\right\}$ defined in (1.8) and (1.7). The results follow by applying the four lemmas above and by choosing $\eta$ in a suitable way (depending, of course by $\eta_{1}, \eta_{2}, \eta_{3}$, where $\eta_{i}, i=1,2,3$ are the exponents defined in the lemmas).

3. Local properties of solutions depending on local properties of the data. A consequence of Theorem 1.1 is a local estimate on solutions of (1.1) away from the singularities of the datum. Indeed assume that $f \in L^{1}(\Omega)$ and there exists a subset $S$ such that $\bar{S} \subset \Omega$ and

$$
f(x)\left[1-\chi_{S}(x)\right] \in L^{m}(\Omega), \quad m>1 .
$$


The existence of a function $\psi \in W^{1, \infty}(\Omega), 0 \leq \psi \leq 1$,

$$
\psi=\left\{\begin{array}{lll}
0 & \text { in } & \bar{S}, \\
1 & \text { in } & \Omega \backslash S^{\prime}
\end{array}\right.
$$

$\bar{S} \subset \subset S^{\prime} \subset \subset \Omega$, follows in a standard way by regularization.

With this choice of $\psi$ we can write the statement of Theorem 1.1 in the following manner.

Theorem 3.1. Assume (1.3), (1.4), (1.5), (3.1) and define $\psi$ as in (3.2). Then there exists a distributional solution $u$ of (1.2) such that

- $\nabla u \in L^{m^{*}}\left(\Omega \backslash S^{\prime}\right)$, if $1<m<\frac{2 N}{N+2}$;

- $\nabla u \in L^{2}\left(\Omega \backslash S^{\prime}\right)$ and $u \in L^{m^{* *}}\left(\Omega \backslash S^{\prime}\right)$, if $\frac{2 N}{N+2} \leq m<\frac{N}{2}$;

- $\nabla u \in L^{2}\left(\Omega \backslash S^{\prime}\right)$ and $u \in L^{\infty}\left(\Omega \backslash S^{\prime}\right)$, if $m>\frac{N}{2}$.

REMARK 3.2. In the previous theorem, the first part of the second item is related with Theorem 2 of [6].

REMARK 3.3. It is possible to state a theorem similar to the previous one if we consider the boundary value problem (1.1). For example, if $S$ is the support of the measure $\mu$, then we can say that $\nabla u \in L^{2}\left(\Omega \backslash S^{\prime}\right)$ and $u \in L^{\infty}\left(\Omega \backslash S^{\prime}\right)$.

We conjecture that in this case $u$ is Hölder continuous in $\Omega \backslash S^{\prime}$. We also conjecture that Neil Trudinger can prove this conjecture (perhaps with the approach of [9]).

Acknowledgements. The authors are grateful to Luigi Orsina and Michaela Porzio for their helpful comments and interest in this work.

\section{REFERENCES}

[1] P. Benilan, L. Boccardo, T. Gallouët, R. Gariepy, M. Pierre and J.L. Vazquez, An $L^{1}$ theory of existence and uniqueness of nonlinear elliptic equations, Ann. Scuola Norm. Sup. Pisa, 22 (1995), pp. 240-273.

[2] L. BocCARdo AND T. GALLOUËT, Nonlinear elliptic and parabolic equations involving measure data, J. Funct. Anal., 87 (1989), pp. 149-169.

[3] L. Boccardo And T. GallouËt, Nonlinear elliptic equations with right hand side measures, Comm. Partial Differential Equations, 17 (1992), pp. 641-655.

[4] L. Bocchrdo, T. GallouËt ANd L. Orsina, Existence and uniqueness of entropy solutions for nonlinear elliptic equations with measure data, Ann. Inst. H. Poincaré, Anal. Non Linéaire, 5 (1996), pp. 539-551.

[5] H. BrEzIS, Equations et inquations non lineaires dans les espaces vectoriels en dualité, Ann. Inst. Fourier (Grenoble), 18 (1968), pp. 115-175.

[6] J. Droniou, Global and local estimates for nonlinear noncoercive elliptic equations with measure data, Comm. Partial Differential Equations, 28 (2003), pp. 129-153.

[7] J. Serrin, Pathological solutions of elliptic differential equations, Ann. Sc. Norm. Sup. Pisa, 18 (1964), pp. 385-387.

[8] G. Stampacchia, Le probléme de Dirichlet pour les quations elliptiques du second ordre coefficients discontinus, Ann. Inst. Fourier, (Grenoble) 15 (1965), pp. 189-258.

[9] N. S. Trudinger, On Harnack type inequalities and their application to quasilinear elliptic equations, Comm. Pure Appl. Math., 20 (1967), pp. 721-747.

[10] N. S. Trudinger, Linear elliptic operators with measurable coefficients, Ann. Scuola Norm. Sup. Pisa, 27 (1973), pp. 265-308. 
L. BOCCARDO AND T. LEONORI 